\title{
Subinhibitory Concentrations of Clinically-Relevant Antimicrobials Affect Resistance-Nodulation- Division Family Promoter Activity in Acinetobacter baumannii
}

\author{
Sonia Prieto Martin Gil, Ana Tajuelo, Mireia López-Siles* and Michael J. McConnell \\ Intrahospital Infections Laboratory, National Center for Microbiology, Instituto de Salud Carlos III (ISCIII), Madrid, Spain
}

OPEN ACCESS

Edited by:

Hanyueh Kuo,

National Taiwan University, Taiwan

Reviewed by:

Bruno Perichon,

Institut Pasteur, France

Jose Ramos-Vivas,

Universidad Europea del Atlántico,

Spain

${ }^{*}$ Correspondence:

Mireia López-Siles

mireia.lopez@isciii.es

mireia.lopezs@udg.edu

Specialty section: This article was submitted to

Evolutionary and Genomic

Microbiology,

a section of the journal

Frontiers in Microbiology

Received: 20 September 2021 Accepted: 11 November 2021 Published: 03 December 2021

Citation:

Prieto Martin Gil S, Tajuelo A, López-Siles M and McConnell MJ (2021) Subinhibitory Concentrations of Clinically-Relevant Antimicrobials Affect Resistance-Nodulation-

Division Family Promoter Activity

in Acinetobacter baumannii.

Front. Microbiol. 12:780201. doi: 10.3389/fmicb.2021.780201
Efflux pumps contribute to multidrug resistance in Acinetobacter baumannii due to their ability to expel a wide variety of structurally unrelated compounds. This study aimed to characterize the effect of subinhibitory concentrations of clinically-relevant antibiotics and disinfectants on the promoter activity of members of the ResistanceNodulation-Division (RND) family in $A$. baumannii. The promoter regions from three RND efflux pumps (AdeABC, AdeFGH and AdelJK) and the AdeRS regulatory system from three different $A$. baumannii strains (ATCC 17961, ATCC 17978, and ATCC 19606) were cloned into a luciferase reporter system (pLPV1Z). Promoter activity was quantitatively assessed in both exponential and stationary phase cultures after exposure to subinhibitory concentrations of four antibiotics from different classes (rifampicin, meropenem, tigecycline and colistin) and two disinfectants (ethanol and chlorhexidine). Subinhibitory concentrations of the compounds tested had variable effects on promoter activity that were highly dependent on the A. baumannii strain, the compound tested and the growth phase. Fold changes in AdeABC promoter activity ranged from 1.97 to 113.7, in AdeFGH from -5.6 to 1.13, in AdelJK from -2.5 to 2, and in AdeRS from -36.2 to -1.32 . Taken together, these results indicate that subinhibitory concentrations of clinically-relevant antibiotics and disinfectants affect the promoter activity of RND family members in $A$. baumannii in a strain and growth phase dependent manner. These results may have important implications for the treatment of infections caused by $A$. baumannii.

Keywords: Acinetobacter baumannii, efflux pumps, RND family, antibiotics, disinfectants, gene expression

\section{INTRODUCTION}

Acinetobacter baumannii is an opportunistic Gram-negative pathogen mainly associated with the healthcare environment, although incidence of infections caused by this microorganism in the community is increasing (Leung et al., 2006; Dijkshoorn et al., 2007; Lin and Lan, 2014). The most common and severe clinical manifestation of this pathogen is hospital-acquired pneumonia associated with mechanical ventilation, although it can cause various pathologies 
such as bacteremia, skin, soft tissue and urinary tract infections (Falagas and Rafailidis, 2007; McConnell et al., 2013). According to the $2017 \mathrm{WHO}$ list of priority pathogens resistant to antibiotics (Taconnelli and Magrini, 2017), A. baumannii is considered within the critical group mainly due to the rapid appearance of multidrug resistant strains (Boucher et al., 2009). Particularly worrisome are descriptions of strains resistant to carbapenems, and to last-line therapeutic options such as colistin (Kyriakidis et al., 2021; Nang et al., 2021). The appearance of these extensively drug resistant strains, for which there are limited treatment options available, has increased the need for understanding the mechanisms underlying acquisition of resistance (Karakonstantis et al., 2020; Kyriakidis et al., 2021).

Although several mechanisms of antimicrobial resistance have been described for A. baumannii (Vila et al., 2007), efflux pumps are of special interest. Firstly, the primitive function of these secretion systems was the removal of metabolic end products or the expulsion of toxins or quorum-sensing molecules (Helling et al., 2002; Sánchez Díaz, 2003), suggesting that these transporters had a different biological function and were later adapted to the expulsion of antibiotics. This confers a non-specificity of substrate and may be related to their ability to actively export multiple, structurally-distinct classes of antimicrobials (Poole, 2002; Sánchez Díaz, 2003). Secondly, these efflux pumps are widely distributed as they have been identified in several microorganisms (Nikaido, 1994) and the presence of several genes coding for different pumps in bacterial genomes have been reported. They thus represent an important intrinsic resistance mechanism. Importantly, efflux pump overexpression has been associated with acquired resistance (Nikaido, 1994; Sánchez Díaz, 2003), which favors the emergence of mutants with a high degree of resistance (Sánchez Díaz, 2003).

Currently, six families of multidrug efflux systems have been associated with multidrug resistance in bacteria (Sánchez Díaz, 2003; Vila et al., 2007; Opazo et al., 2009), and at least five of them have been found in the genome of A. baumannii (Coyne et al., 2011). Among them, overexpression of the resistance-nodulation-division (RND) family, which includes pumps that are proton antiporters, has been associated with the development of multidrug-resistance in this species (Yoon et al., 2013; Salehi et al., 2021). All RND efflux pumps are a three-component based system composed of a transporter protein located in the cytoplasmic membrane, a membrane fusion protein in the periplasm and an outer membrane protein or porin (OMP) that is located in the outer membrane (Wieczorek et al., 2008). In A. baumannii, the RND family efflux pumps that have been identified include AdeABC, AdeIJK, AdeFGH, AdeDE and AdeXYZ. Whereas the former appears to be unique to A. baumannii species, AdeDE and AdeXYZ pumps have been found in other Acinetobacter species (Chu et al., 2006; Fournier et al., 2006). Regulatory systems have been described for some of these pumps such as the two-component AdeRS that is encoded upstream of adeABC (Marchand et al., 2004), AdeN a TetR transcriptional regulator which regulates AdeIJK (Rosenfeld et al., 2012) and AdeL, a LysR-Type regulator that overlaps with and regulates adeFGH (Coyne et al., 2010b).
Resistance-nodulation-division members have been associated with resistance to several antibiotics, but the environmental stimuli that lead to their activation/inhibition are not fully understood. Several studies have evaluated the contribution of efflux pumps in A. baumannii to resistance to a given compound by gene inactivation experiments or the use of efflux pump inhibitors (Magnet et al., 2001; Damier-Piolle et al., 2008; Coyne et al., 2010b; Rajamohan et al., 2010). However, few studies have characterized how pump expression varies as a consequence of exposure to subinhibitory concentrations of antimicrobials. In addition, while data exist with respect to several antibiotics, information regarding the effect of disinfectants is limited. Furthermore, most of these studies have focused on multidrug resistant strains (Ardebili et al., 2014; Lari et al., 2018) and the effect of antimicrobials on the expression of these pumps on non-resistant strains remains to be fully characterized, an aspect which could shed light on the emergence of resistance.

The aim of this study is to characterize how subinhibitory concentrations of antimicrobials affect the expression of members of the RND family in different A. baumannii strains. To achieve this, we studied the promoters that control the expression of the operons of three efflux pumps of the RND family (AdeABC, AdeFGH, and AdeIJK) and the twocomponent regulatory system (AdeRS) to explore changes in their expression. We employ a previously-validated plasmid reporter system that has been used for characterization of spatiotemporal gene expression dynamics in A. baumannii under multiple experimental conditions including exposure to UV light, chemical/disinfectant treatment, and iron depletion (Lucidi et al., 2019). This system was developed specifically for quantifying the activity of Acinetobacter promoters. We studied the effect of antibiotics with different mechanisms of action and disinfectants often used in the clinical setting, to cover the wide spectrum of substrates that can be released by this expulsion system.

\section{MATERIALS AND METHODS}

\section{Bacterial Strains and Culture Media}

Acinetobacter baumannii ATCC $^{\circledR} 17978^{\mathrm{TM}}$ (ATCC ${ }^{\circledR}$ 17978), ATCC $^{\circledR} 19606^{\text {TM }}\left(\right.$ ATCC $\left.^{\circledR} 19606^{T}\right)$ and ATCC ${ }^{\circledR} 17961^{\text {TM }}\left(\right.$ ATCC $^{\circledR}$ 17961) were used for expression assays in order to cover different genetic backgrounds. Escherichia coli TOP10 was used as an intermediate host for all plasmid constructs. E. coli ATCC $^{\circledR} 25922^{\mathrm{TM}}$, Staphylococcus aureus ATCC $^{\circledR} 29213^{\mathrm{TM}}$ and Pseudomonas aeruginosa ATCC $^{\circledR} 27853^{\mathrm{TM}}$ were used as control strains in minimum inhibitory concentration (MIC) experiments according to guidelines by Clinical and Laboratory-Standards Institute for antimicrobials (CLSI, 2017). All bacterial strains were grown routinely at $37^{\circ} \mathrm{C}$ in Luria-Bertani broth (LB) or on LB agar plates (Condalab) and supplemented with gentamicin at an appropriate concentration $(10-100 \mu \mathrm{g} / \mathrm{ml})$ for transformants selection. Mueller-Hinton broth II (MHB-II, Sigma) was used for growth in both MIC and luminescence assays. For long-term storage, bacterial cultures were grown on LB supplemented with $20 \%$ glycerol and stored at $-80^{\circ} \mathrm{C}$ for further use. 


\section{Susceptibility Testing}

Minimum inhibitory concentration values were determined for a representative compound from different families of antimicrobials and disinfectants, covering a variety of mechanisms of action, and that included: colistin (COL), meropenem (MEM), rifampicin (RIF), tigecycline (TGC), chlorhexidine (CLX), and ethanol (EtOH). Product references and concentrations used are provided in Supplementary Table 1. Overnight cultures of each strain were adjusted to $\mathrm{OD}_{600}=0.1$ in MHB-II and the MIC was determined according to the recommendations of the Clinical and Laboratory Standards Institute for antimicrobials and the Sociedad Española de Enfermedades Infecciosas y Microbiología Clínica (Picazo, 2000; CLSI, 2017). For each strain, MIC values were determined at least by triplicate on three different days.

\section{Preparation of Electrocompetent Cells and Electroporation Conditions}

Competent E. coli TOP10 cells were prepared for electrotransformation as follows. E. coli TOP10 cells were grown in $\mathrm{LB}$ at $37^{\circ} \mathrm{C}$ for $16 \mathrm{~h}$. Cultures were refreshed by dilution 1:100 in $100 \mathrm{~mL}$ of $\mathrm{LB}$ and incubated for $3-4 \mathrm{~h}$ at $37^{\circ} \mathrm{C}$ with shaking at $200 \mathrm{rpm}$, until the $\mathrm{OD}_{600}$ reached $\sim 0.5$. Cells were harvested by centrifugation (6,000 rpm for $10 \mathrm{~min})$, washed four times with cold $10 \%$ glycerol, and suspended in $2 \mathrm{~mL}$ of $10 \%$ glycerol. One hundred-microliter aliquots of competent cells were stored at $-80^{\circ} \mathrm{C}$ until further use.

A. baumannii electrocompetent cells were prepared as reported elsewhere (Lucidi et al., 2019) with minor modifications. Briefly, bacteria were grown in $10 \mathrm{~mL}$ of $\mathrm{LB}$ at $37^{\circ} \mathrm{C}$ for $16 \mathrm{~h}$. Bacterial cultures were diluted 1:100 into $100 \mathrm{~mL}$ of $\mathrm{LB}$ and the cells were grown for $24 \mathrm{~h}$ at $37^{\circ} \mathrm{C}$ with shaking at $200 \mathrm{rpm}$. Cells were harvested and washed as indicated above. Eightymicroliter aliquots of competent cells were stored at $-80^{\circ} \mathrm{C}$ until further use.

Electroporation was performed using one aliquot of competent cells and 100-250 ng of plasmid in electroporation cuvettes of $0.1 \mathrm{~cm}$ electrode gap (Bio-Rad). After pulsing with Bacteria default settings $(1.8 \mathrm{kv}, \geq 3.9 \mathrm{mS})$, the cells were recovered in $1 \mathrm{~mL}$ of $\mathrm{LB}$ for $1 \mathrm{~h}$ at $37^{\circ} \mathrm{C}$ with shaking at $200 \mathrm{rpm}$ and then transformants were selected on LB agar plates with the appropriate gentamicin concentration.

\section{Primer Design and Amplification of the Promoter Region}

To carry out gene expression experiments, the operons corresponding to the adeABC, adeFGH, adeIJK efflux pumps and the adeRS control system were located in the genome of the A. baumannii ATCC $19606^{T}$ strain (Accession number: $\mathrm{CP} 046654)$. The $500 \mathrm{bp}$ upstream of the start codon of the first gene in the operon were selected. Primers (Table 1) were designed from the first and last 20 nucleotides of the 500 bp sequence, and the target sequences of BamHI and NotI were included at the $5^{\prime}$ end of the forward and reverse primers, respectively. As the promoter regions of the four genes were highly similar among strains ( $\geq 98 \%$, Supplementary Figure 1) one set of primers was used to amplify the $500 \mathrm{bp}$ fragment in all the A. baumannii strains studied. All primers were obtained with desalted purification (Sigma).

Genomic DNA was extracted with the freeze/thaw method and further used for promoter amplification through conventional PCR. The reaction mixture was composed of: $1 \times$ EconoTaq PLUS 2X Master Mix (Sigma-Aldrich), $1 \mu \mathrm{M}$ of forward primer, $1 \mu \mathrm{M}$ of reverse primer and $1 \mu \mathrm{L}$ of genomic DNA extract as template, in a total volume of $50 \mu \mathrm{L}$.

PCR conditions consisted of $10 \mathrm{~min}$ at $95^{\circ} \mathrm{C}$ for initial denaturation, followed by 30 cycles of $30 \mathrm{~s}$ at $95^{\circ} \mathrm{C}, 30 \mathrm{~s}$ at $58^{\circ} \mathrm{C}$, and $30 \mathrm{~s}$ at $72^{\circ} \mathrm{C}$ and then a final extension of $10 \mathrm{~min}$ at $72^{\circ} \mathrm{C}$. Products were visualized under UV light after gel electrophoresis on $1 \%(w t / v o l)$ agarose gels in $1 \times$ TAE buffer (Tris-acetateEDTA, pH 8.0) stained with $1 \times$ Gel Red 10,000 $\times$ (Biotium). The bands corresponding to the promoters were excised and the DNA was purified using the NucleoSpin Gel and PCR Clean-up kit (Macherey-Nagel) according to manufacturer recommendations.

\section{Plasmid Construction and Cloning Into Acinetobacter baumannii Strains}

The pLPV1Z shuttle-vector, which includes the luciferase reporter systems (Lucidi et al., 2019), was used to evaluate changes in gene expression. A total of 12 plasmids were constructed including the promoters of the four operons, for the three A. baumannii strains of study. Both, the plasmid and the purified $500 \mathrm{bp}$ amplicon including the promoters were doubledigested with Bam HI and NotI (New England Biolabs) for $30 \mathrm{~min}$ and $37^{\circ} \mathrm{C}$. After purification using the NucleoSpin Gel and PCR Clean-up kit (Macherey-Nagel) according to manufacturer recommendations, promoter and vector were ligated with T4 DNA ligase (New England Biolabs) at a plasmid:insert ratio of 1:20-1:50 at $16^{\circ} \mathrm{C}$ for $20 \mathrm{~h}$. The final plasmid constructions were used to transform electrocompetent E. coli TOP10 cells. Transformants were selected by plating on LB agar supplemented with gentamicin $(50 \mu \mathrm{g} / \mathrm{ml})$ and screened by conventional PCR with T3 and RBS primers (Table 1), performed as described above.

Verified plasmid constructs were extracted and purified from a $10 \mathrm{~mL}$ E. coli TOP10 culture in LB supplemented with gentamicin $50 \mu \mathrm{g} / \mathrm{mL}$ using the NucleSpin Plasmid kit (Macherey-Nagel) following the protocol recommended by the manufacturer, and further confirmed by sequencing. Subsequently, constructs were introduced into electrocompetent cells of the corresponding strain of $A$. baumannii by electroporation. The clones incorporating the plasmid were selected by gentamicin resistance (10-100 $\mu \mathrm{g} / \mathrm{mL}$ ) according to the requirements of each strain (Table 2). Transformants were screened by conventional PCR with T3 and RBS primers as indicated previously, and also by measuring bioluminescence emission of the luciferase-luciferin system in the Orion II Microplate Luminometer (Berthold).

\section{Qualitative Analysis of Promoter Activity}

Agar diffusion assays were used to visually assess the effect of subinhibitory concentrations of antimicrobials on the expression of the operons. Briefly, cultures were prepared as described 
TABLE 1 | Primers used in this study and PCR conditions.

\begin{tabular}{|c|c|c|c|c|c|}
\hline Target & Primer name & Sequence $\left(5^{\prime}-3^{\prime}\right)^{a}$ & Use & $\mathrm{Tm}^{b}$ & Reference \\
\hline \multirow[t]{2}{*}{ ade $A B C$} & P_adeA_F & ATTCGGGATCCCTTCATTTGGGTTAAAAGGCTTC & Promoter amplification & 59.67 & This study \\
\hline & P_adeA_R & ATTCGGCGGCCGCCATACTGTCCAAACCTAGTGAGTT & & 57.02 & This study \\
\hline \multirow[t]{2}{*}{ adeFGH } & P_adeF_F & ATTCGGGATCCCGATACAGGCACATCAATACGA & Promoter amplification & 58.77 & This study \\
\hline & P_adeF_R & ATTCGGCGGCCGCAGGTGCTCCTAGTTATITGGATACC & & 59.85 & This study \\
\hline \multirow[t]{2}{*}{ adelJK } & P_adel_F & ATTCGGGATCCTTGCACGCGTAGGCGG & Promoter amplification & 60.02 & This study \\
\hline & P_adel_R & ATTCGGCGGCCGCGTTCCACCTCGTIAGATAAAATCTA & & 58.33 & This study \\
\hline \multirow[t]{2}{*}{ adeRS } & P_adeR_F & ATTCGGGATCCCTITGAGTCTTGCTACCTCAGCTT & Promoter amplification & 59.53 & This study \\
\hline & P_adeR_R & ATTCGGCGGCCGCGATAATCTGGCTATAGAAAGTGCTTC & & 58.13 & This study \\
\hline \multirow[t]{2}{*}{ pLPV1Z } & T3 (modified) & GCAATTAACCCTCACTAAAGG & Constructs confirmation & 54.09 & Lucidi et al., 2019 \\
\hline & RBS & СТТААТТСТССТСТТАСТТАСТТ & & 51.46 & This study \\
\hline
\end{tabular}

${ }^{a}$ Boldface target of the BamHl enzyme in forward (F) primers and of the Notl enzyme in reverse (R) primers, respectively.

Italics nucleotides indicate a random sequence included to facilitate the action of the enzyme.

${ }^{b}$ To calculate Tm, additional nucleotides to facilitate cleavage by restriction enzymes were not included.

above, with a culture adjusted to $\mathrm{OD}_{600}=0.1$ to inoculate Mueller-Hinton agar plates (Oxoid) by swabbing. Up to $50 \mu \mathrm{L}$ of the appropriate antimicrobial solution ranging from $25 \times$ to $40,000 \times$ of MIC (Supplementary Table 1) was placed in the center of the plate and incubated overnight at $37^{\circ} \mathrm{C}$. A control with water was included as growth control and to assess the basal expression of the promoters in the absence of antimicrobials. Parental strains without any construct were also tested with all the antimicrobials as negative controls for bioluminescence. Bioluminescence was visualized using an IVIS Lumina XRMS series II in vivo Imaging System (Perkin Elmer) setting $1 \mathrm{~s}$ as exposure time for image capture.

\section{Quantitative Analysis of Promoter Activity}

Initial experiments were carried out to optimize inoculum and antimicrobial concentration, as well as the most appropriate time for measurement (data not shown). The optimized protocol

TABLE 2 | Minimum inhibitory concentration $(\mu \mathrm{g} / \mathrm{mL})$ for $A$. baumannii strains ATCC 17978, ATCC $19606^{T}$ and ATCC 17961.

\begin{tabular}{|c|c|c|c|c|}
\hline \multirow[b]{2}{*}{ Antimicrobia } & & \multicolumn{3}{|c|}{ A. baumannii strain } \\
\hline & & ATCC 17978 & ATCC $19606^{T}$ & ATCC 17961 \\
\hline \multicolumn{5}{|l|}{ Disinfectant } \\
\hline & Ethanol & 6.25 & 3.13 & 3.13 \\
\hline & Chlorhexidine & 16 & 16 & 16 \\
\hline \multicolumn{5}{|l|}{ Antibiotic } \\
\hline & Gentamicin* & 1 & 8 & 1.5 \\
\hline & Colistin & 0.5 & 0.5 & 0.25 \\
\hline & Meropenem & 0.25 & 1 & 0.5 \\
\hline & Rifampicin ${ }^{\star \star}$ & 4 & 2 & 4 \\
\hline & Tigecycline & 0.5 & 1 & 0.5 \\
\hline
\end{tabular}

*MIC value determined by E-test strips.

${ }^{* *}$ At least one of the control strains featured values not within the range established by the CLSI.

The strains E. coli ATCC 25922, S. aureus ATCC 27853 and P. aeruginosa ATCC 29213 were used as controls and MIC values were within the expected range. was used in the subsequent experiments to establish promoter activity. Briefly, A. baumannii strains carrying their respective construct of pLPV1Z:PadeA, pLPV1Z:PadeF, pLPV1Z:PadeR and pLPV1Z:PadeI as well as the parental strain without plasmid were grown overnight at $37^{\circ} \mathrm{C}$ on $\mathrm{LB}$ agar with the appropriate gentamicin concentration. A subculture in LB maintaining the previous gentamicin concentration was incubated at $37^{\circ} \mathrm{C}$ for $20 \mathrm{~h}$. These cultures were adjusted to an $\mathrm{OD}_{600}=0.1$ and then diluted 100 -fold to inoculate $5 \mathrm{~mL}$ of MHB-II supplemented with the appropriate antimicrobial, at a concentration corresponding to $1 / 4$ of the MIC (Supplementary Table 1). A growth control containing media without antimicrobial was included in all the experiments and was used for subsequent data normalization. To calculate background, a sample consisting of LB supplemented with antimicrobial was also included. The $\mathrm{OD}_{600}$ value and bioluminescence emission were recorded after incubation for $6 \mathrm{~h}$ (exponential phase) and $24 \mathrm{~h}$ (stationary phase) at $37^{\circ} \mathrm{C}$ and continuous shaking at $200 \mathrm{rpm}$. Cultures were grown in an incubator, and a $100 \mu \mathrm{l}$ sample was taken at those time intervals to quantify $\mathrm{OD}_{600}$ on the one hand and bioluminescence on the other hand, using the Tecan Sunrise Absorbance Reader Analyser (Tecan) and an Orion II Microplate Luminometer (Berthold) reader, respectively. All experiments were performed at least in quadruplicate on four different days.

\section{Statistical Analysis}

To account for differences in growth between strains and conditions, bioluminescence was normalized to the $\mathrm{OD}_{600}$ of the culture prior to fold-change calculation. To calculate the activity of the promoters, the normalized data was divided by that obtained from the corresponding growth control (culture without antimicrobial) in order to obtain the fold-change. For fold-change $<1$ the inverse was calculated to obtain the real magnitude of the change and was expressed as negative growth in order to indicate decreased expression. In some cases, it was not possible to calculate the fold change of the strains because it did not grow sufficiently $\left(\mathrm{OD}_{600}<0.02\right)$, and/or no growth was recorded after $24 \mathrm{~h}$ of incubation. 
First, we determined if the fold-change calculated was different from 1 (as we established 1 as no changes in expression) by a one sample T-test. Secondly, the Kruskal-Wallis test was used to calculate differences in expression between strains and compounds. Further pairwise comparisons by subcategories of these variables were analyzed using the Dunn's multiple comparison test. In this case, non-parametric statistical tests were used given the non-normal distribution of the data and the lack of homoscedasticity as assessed by Kolmogorov-Smirnov and Levene tests, respectively. Finally, correlation between expression was assessed by strain by calculating the Pearson correlation coefficient. In this case, the mean values of fold-changes of the four replicates performed in each antimicrobial treatment were used. Graphpad (Prism) and SPSS (IBM) were used for data plotting and statistical analysis. A $p$-value $\leq 0.05$ was considered significant.

\section{RESULTS}

\section{Susceptibility to Antimicrobials}

All three strains of $A$. baumannii were sensitive to the antimicrobials used according to CLSI and EUCAST breakpoint tables for interpretation of MIC values (CLSI, 2012; The European Committee on Antimicrobial Susceptibility Testing, 2021). However, each strain featured a different susceptibility profile (Table 2). A. baumannii ATCC 17961 had the lowest MIC for COL, whereas strain ATCC 17878 was the most sensitive to MEM. The A. baumannii ATCC $19606^{T}$ MIC of RIF was the lowest among the three strains tested. MIC values ranged between $0.25-16 \mu \mathrm{g} / \mathrm{mL}$, and variation in the range of twoto fourfold were observed between strains. With regard to disinfectants, A. baumannii ATCC 17978 demonstrated a higher MIC for $\mathrm{EtOH}$, which was twofold that of the other strains (Table 2). In contrast, the CLX MIC was similar for all the A. baumannii strains tested.

\section{Qualitative Analysis of Expression}

We first aimed to characterize the bioluminescence produced by the different plasmid constructs when subjected to a concentration gradient of the different compounds to determine if there were qualitative differences in the expression from the RND promoters at subinhibitory concentrations of antimicrobials (Figures 1A,B).

In strains containing the ade $A B C$ promoter construct, a halo of bioluminescence was observed at subinhibitory concentrations of all the antimicrobials tested in strains ATCC 17978 and ATCC $19606^{T}$. In contrast, for strain ATCC 17961 the halo of bioluminescence was only observed in the presence of CLX and MEM.

In the case of the adeFGH promoter, all strains featured a rather similar pattern. No bioluminescence was observed for any of the antimicrobials.

With regard to expression of adeIJK promoter, assays with water revealed a low basal expression in the case of ATCC 17978 and ATCC 17961 strains but not in ATCC $19606^{T}$. A halo of increased bioluminescence was observed at subinhibitory concentrations in the presence of all the antimicrobials for ATCC 17978. This general phenomenon was also observed in the other two strains, but no bioluminescence was detected for strain ATCC $19606^{T}$ in the presence of CLX. For strain ATCC 17961 , in assays with CLX only baseline levels of bioluminescence were observed. Altogether this result suggests a strain-specific response of this promoter to certain compounds.

Constitutive basal expression of adeRS promoters was also revealed in the absence of antimicrobials, especially for ATCC $19606^{T}$ and ATCC 17961. Nonetheless, halos of increased bioluminescence were observed with subinhibitory concentrations of all antimicrobials in all strains. This was especially clear in the presence of MEM in ATCC $19606^{T}$. These antibiotics also induced differences in expression in adeABC, suggesting that they may affect the expression of both the efflux pump and the regulatory system.

\section{Quantitative Analysis of Expression}

To further study the effect of subinhibitory concentrations of antimicrobials on the expression of RND family members, quantitative assays were carried out by determining luciferase activity of the cultures treated with the different antimicrobials at $1 / 4$ of the MIC. This allowed us to calculate fold changes as well as to determine whether there was overexpression or decreased expression of the promoter compared to growth in absence of antimicrobials.

Significant overexpression of ade $A B C$ was observed for $\mathrm{EtOH}$ $(p=0.023)$, CLX $(p=0.0001)$ and $\operatorname{RIF}(p=0.046)$ in strain ATCC 17978 (Figure 2). It is remarkable that CLX caused over a 100-fold-change at the exponential phase of growth. This effect was still significant, although smaller, during the stationary phase ( $p=0.039)$. CLX also caused a 45 -fold increase in expression of this promoter in strain ATCC 17961 during the stationary phase $(p=0.047)$. None of the compounds tested had a significant effect on the expression of the adeABC promoter in strain ATCC 19606 during exponential and stationary growth.

For adeFGH promoter expression, almost all fold-change values were around 1 or below (Figure 3). This suggested no overexpression from these promoters at subinhibitory concentrations of the antimicrobials and was in line with the results observed in the qualitative assay showing no bioluminescence. Only EtOH $(p=0.001)$ showed significantly decreased expression during the stationary phase in strain ATCC 17978. For strains ATCC $19606^{T}$ and ATCC 17961, significant effect of the antimicrobials was observed only during exponential phase. EtOH $(p=0.0001)$ and MEM $(p=0.026)$ significantly decreased the expression of adeFGH in strain ATCC $19606^{T}$. For strain ATCC 17961, MEM and of TGC had a significant effect on the expression from this promoter, however, they had opposite effects on promoter activity. Whereas MEM caused a 1.125-fold overexpression $(p=0.034)$, TGC caused a 1.25 -fold decrease in expression $(p=0.0001)$.

With regards to expression from the adeIJK promoter (Figure 4), the effect on expression was mainly observed during the exponential phase. In strain ATCC $19606^{T}$, CLX, COL and MEM significantly decreased the expression of this promoter $(p \leq 0.0001)$. For strain ATCC 17978, EtOH $(p=0.0001)$, 


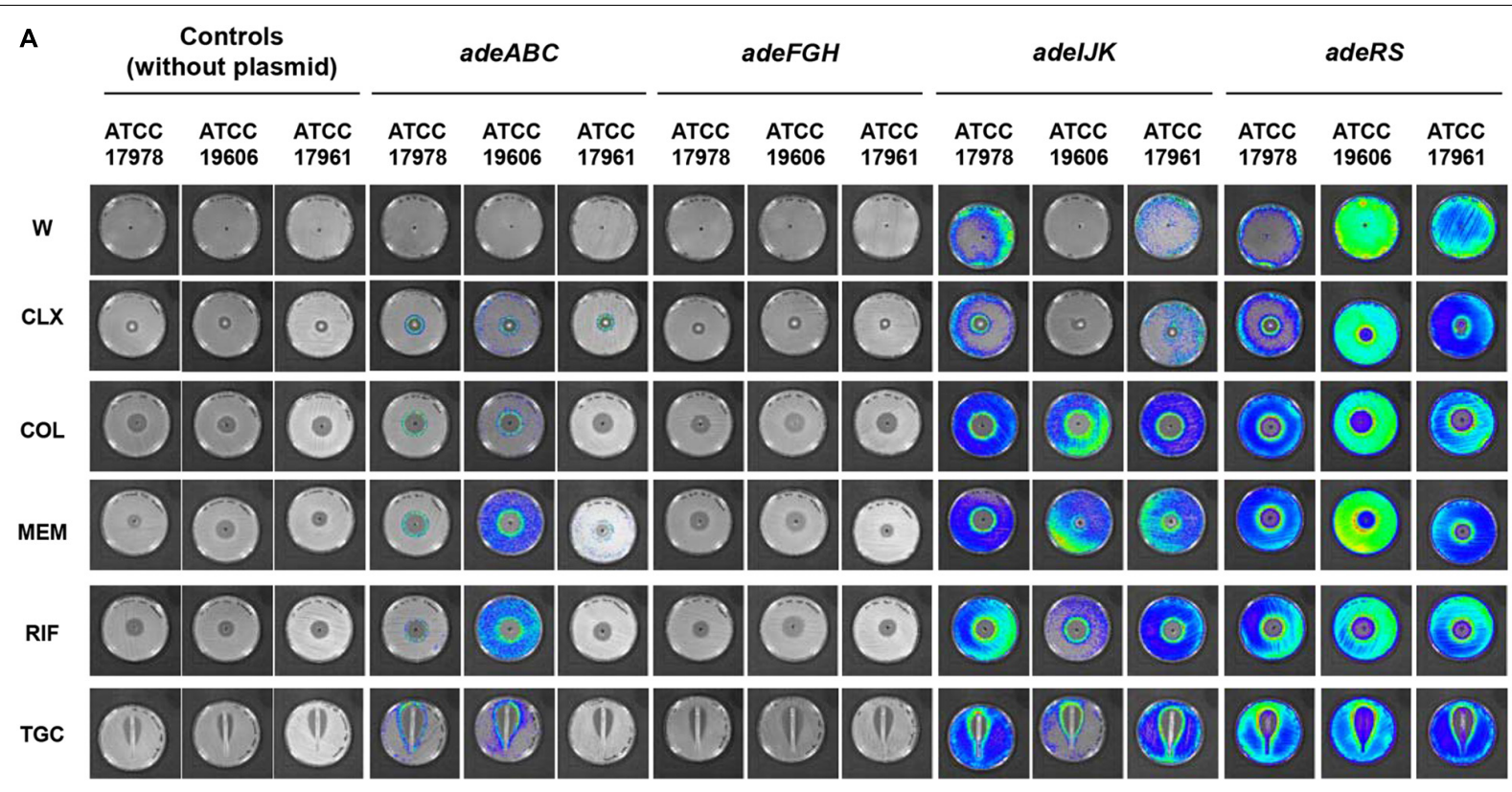

W: Water; CLX: Clorhexidine, COL: Colistine, MEM: Meropenem, RIF: Rifampicin, TGC: Tigecyclin
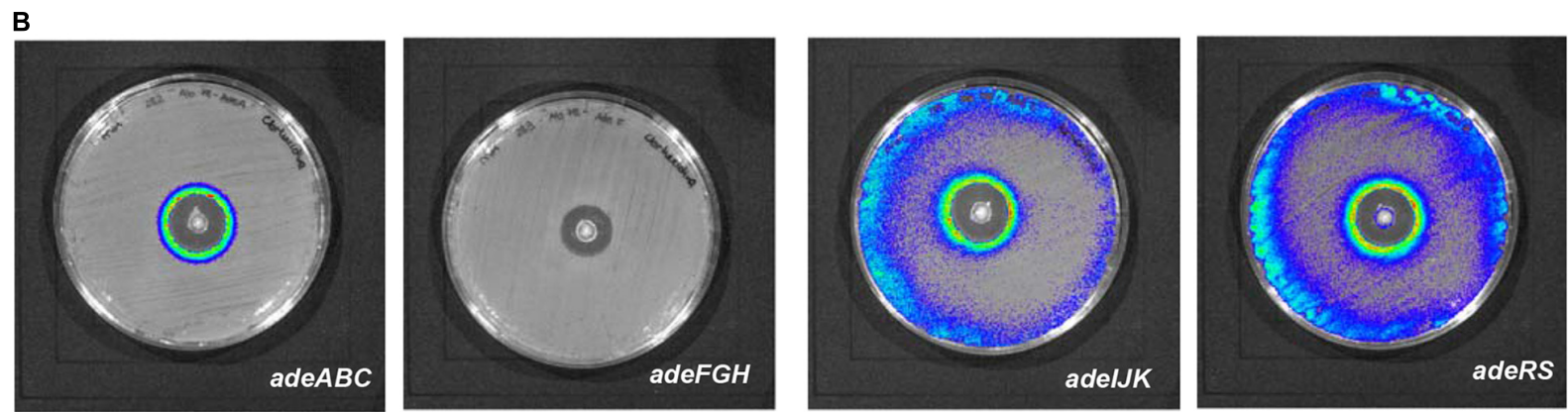

FIGURE 1 | Qualitative analysis of promoter activity in the 12 plasmid constructions. (A) Bioluminescence emission of the different constructions when subjected to a concentration gradient of different compounds for all promoters. (B) Magnification of chlorhexidine treatments in strain ATCC17978 for adeABC, adeFGH, adelJK, adeRS (from left to right). Luminescence intensity is represented by color from greatest to least as follows: red, yellow, green and blue.

CLX $(p=0.006)$ and TGC $(p=0.0001)$ also showed a significant downregulation. Interestingly, RIF caused a significant overexpression of this pump in all strains $(p \leq 0.025)$, ranging between 1.44 to 2 -fold increase in expression. In the stationary phase, only a significant decreased expression was observed for TGC in strain ATCC 17978 ( $p=0.0001)$.

For the adeRS promoter (Figure 5), EtOH, CLX, and MEM significantly decreased the expression from this promoter $(p \leq 0.026)$ during the exponential phase for strain ATCC $19606^{T}$. The same effect was observed for CLX in strain ATCC $17978(p=0.032)$. No significant effect on the stationary phase was observed for any of the antimicrobials in the other two strains.

It is important to point out that the apparent inconsistencies between qualitative (Figure 1) and quantitative assays (Figures 2-5) are due to technical limitations of the qualitative assay, related to the detection limit. This assay represents an end-point picture where overexpression of promoters can be observed through increases in luminescent intensity. In these images, no changes in expression or reduced expression would be observed as no bioluminescence. In contrast, in the quantitative assay, by comparison with the control without antimicrobial, a decrease in expression can also be calculated. This is the case of adeFGH, for example, where quantitative analysis demonstrated that almost all fold-change values were around 1 or below, and therefore not detected in the qualitative assay.

\section{Strain-Specificity of Promoter Activity}

Data from the quantitative expression assays were used to assess if the tested compounds affected the expression of the four promoters similarly in the three A. baumannii strains used in this study. Altogether, the result of this analysis showed differences among strains in promotors expression under exposure to 


\section{Exponential phase}
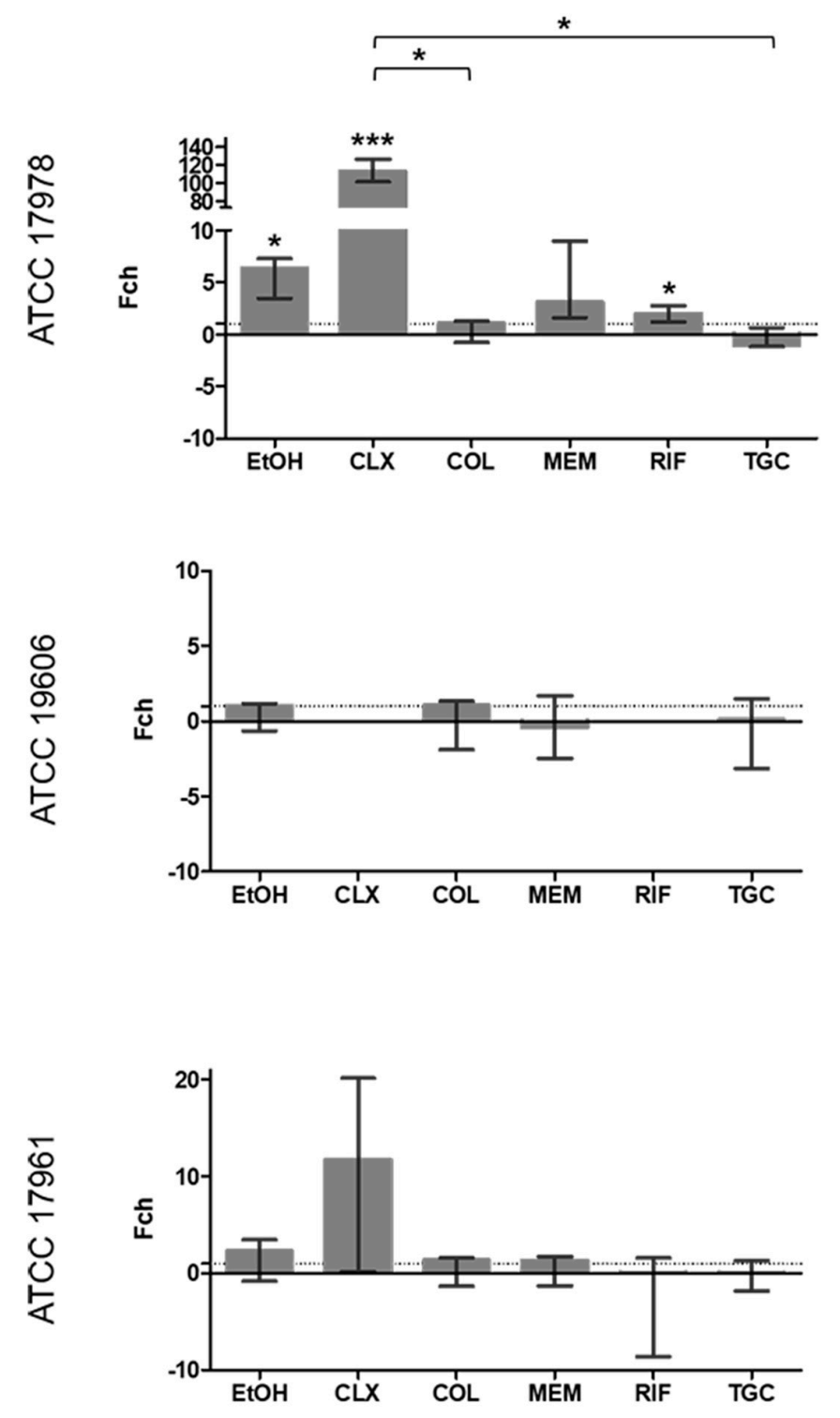

Stationary phase
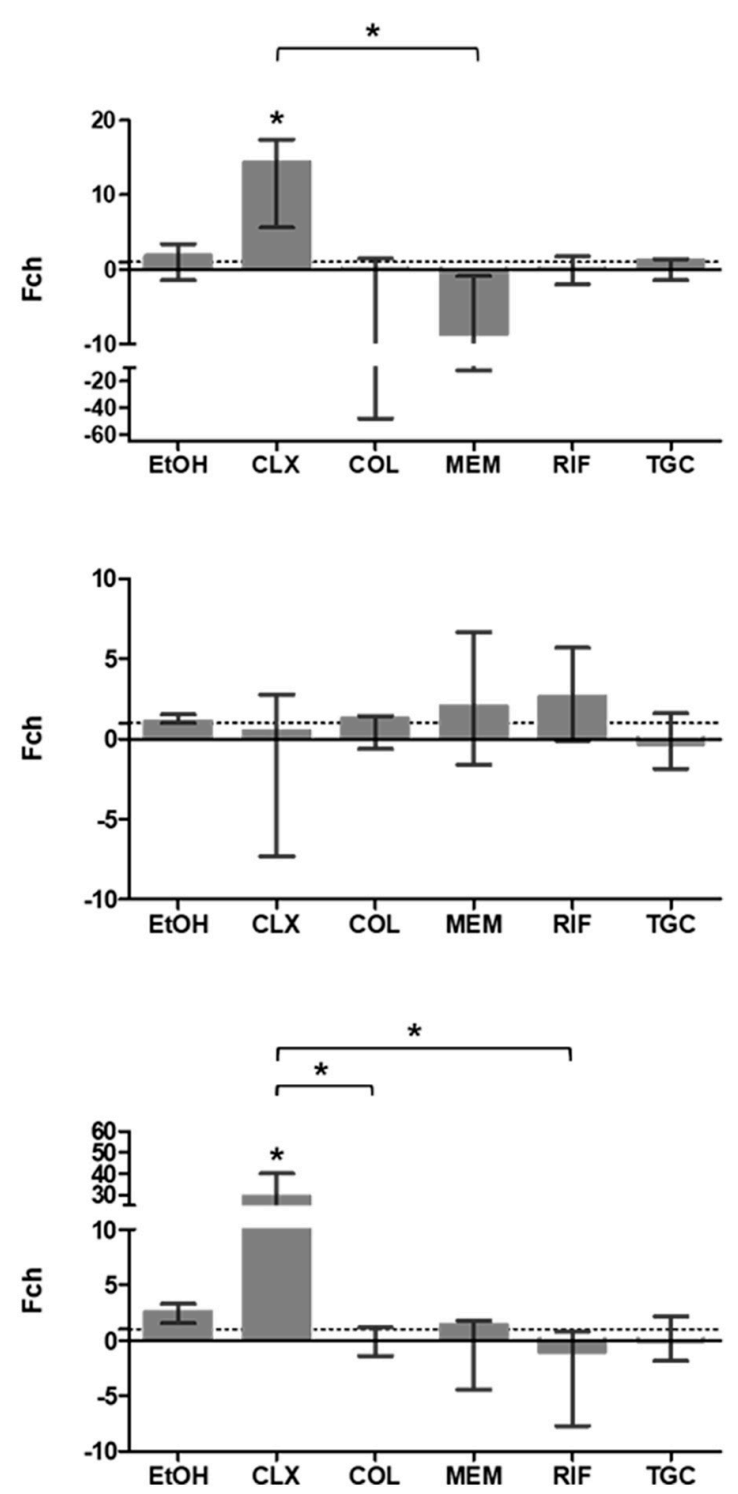

FIGURE 2 | Fold change in adeABC promoter activity in the three strains included in the study calculated at $6 \mathrm{~h}$ (exponential phase) and $24 \mathrm{~h}$ (stationary phase) in the presence of subinhibitory concentrations of antibiotics. The fold-change represents the difference in luminescence. Values $>1$ are considered as increased expression and values $<1$ as decreased expression. Values = 1 represent no changes in expression. There is no representation of groups with no growth $\left(\mathrm{OD}_{600}<0.02\right)$. Brackets indicate significant differences between treatments; ${ }^{\star} p<0.05$, and ${ }^{\star \star \star} p<0.001$. Colistin (COL), meropenem (MEM), rifampicin (RIF), tigecycline (TGC), chlorhexidine (CLX), and ethanol (EtOH).

certain compounds and also depending on growth phase of the culture (Figure 6).

For the adeABC promoter, significant differences between strains were observed in four out of the six antimicrobials tested. During the exponential growth phase, differences in the effect of EtOH and CLX were observed ( $p \leq 0.03$ ). In the stationary phase, significant differences were also obtained for $\operatorname{CLX}(p=0.015)$ and MEM $(p=0.04)$. Two antimicrobials affected
adeFGH promoter differently between strains. In this case, during exponential phase significant differences were found for CLX and MEM $(p=0.02)$. For the adeIJK promoter, no statistically significant differences were observed between strains with any of the compounds tested, regardless of growth phase. For the adeRS promoter, significant differences were observed with CLX and MEM treatments $(p \leq 0.03)$ in the exponential phase. In the stationary phase there were also significant differences after 


\section{Exponential phase}
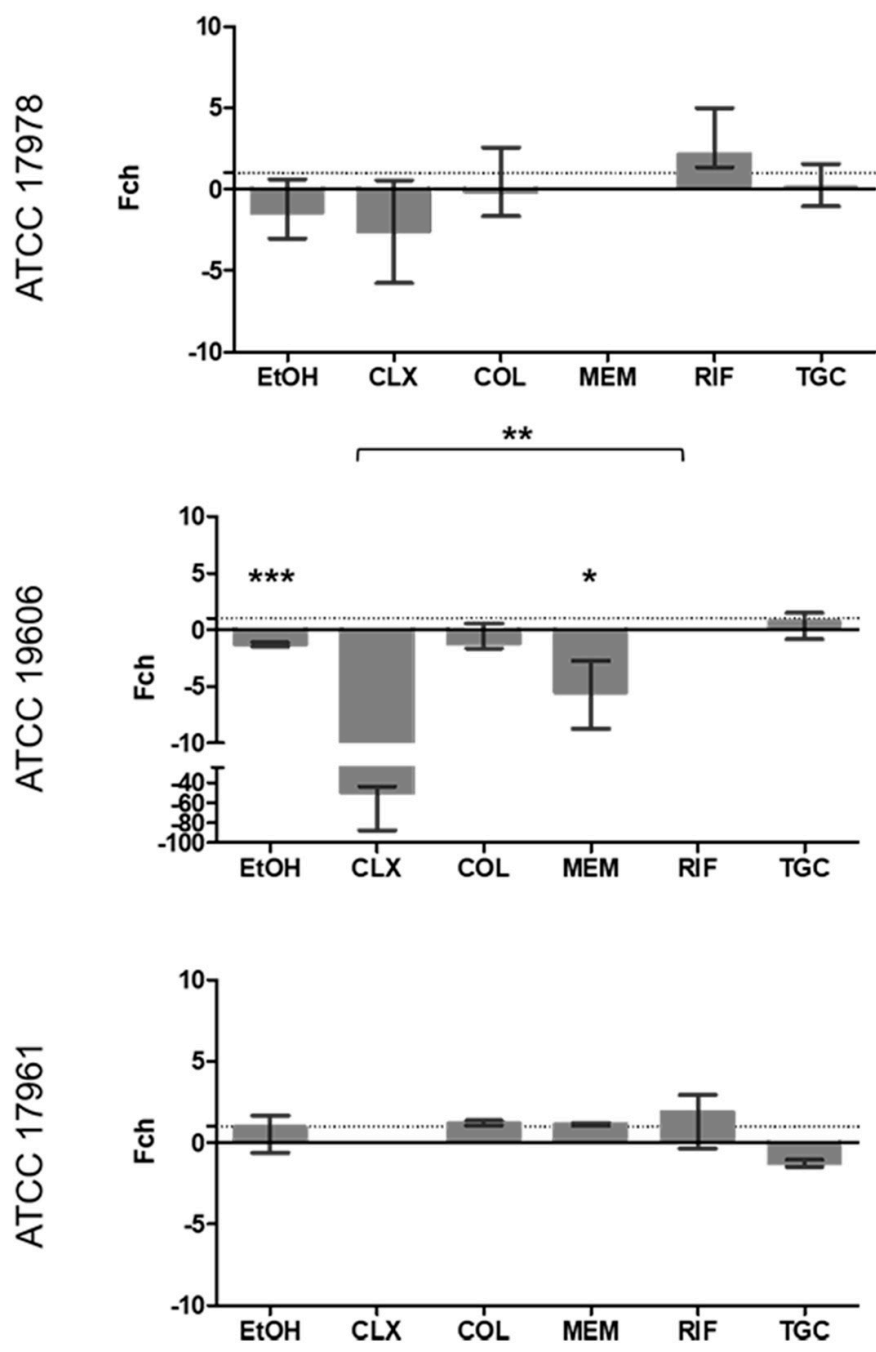

Stationary phase
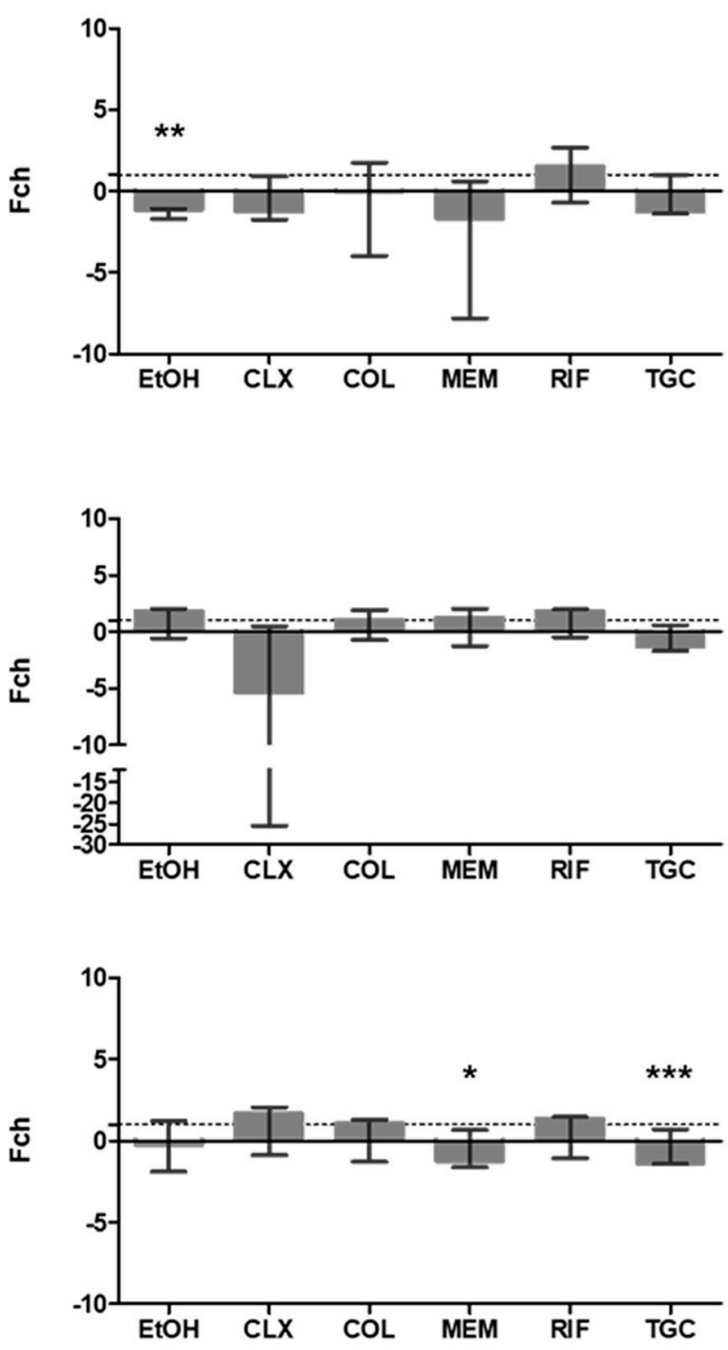

FIGURE 3 | Fold change in adeFGH promoter activity in the three strains included in the study calculated at $6 \mathrm{~h}$ (exponential phase) and $24 \mathrm{~h}$ (stationary phase) in the presence of subinhibitory concentrations of antibiotics. The Fold-change represents the difference in luminescence. Values $>1$ are considered as increased expression and values $<1$ as decreased expression. Values = 1 represent no changes in gene expression. There is no representation of groups with no growth $\left(\mathrm{OD}_{600}<0.02\right)$. Brackets indicate significant differences between treatments; ${ }^{\star} p<0.05,{ }^{\star *} p<0.01$ and ${ }^{* \star *} p<0.001$. Colistin $(\mathrm{COL})$, meropenem (MEM), rifampicin (RIF), tigecycline (TGC), chlorhexidine (CLX), and ethanol (EtOH).

treatment with CLX and RIF $(p \leq 0.024)$. Overall, CLX was the compound that featured more differences in expression among strains, regardless of the growth phase.

\section{Correlation Between Promoter Expression and Strain}

To estimate if the expression of the different RND family members were related, based on the strain tested, for each strain we calculated pairwise correlations of the mean fold-changes obtained with all the antimicrobials tested together (Table 3). In general, few significant correlations were observed. During the exponential phase, negative correlation between adeABC and adeRS was found in strain ATCC 17978 whereas positive correlation between adeFGH and adeRS was found in strain ATCC $19606^{T}$. In the stationary phase, positive correlation between adeIJK with adeABC and adeRS were found in strains ATCC $19606^{T}$ and ATCC 17961, respectively.

\section{DISCUSSION}

Efflux pumps contribute to multidrug resistance in A. baumannii due to their ability to expel a wide variety of structurally unrelated compounds. In this study we analyzed the effect of subinhibitory concentrations of antimicrobials on the expression 


\section{Exponential phase}
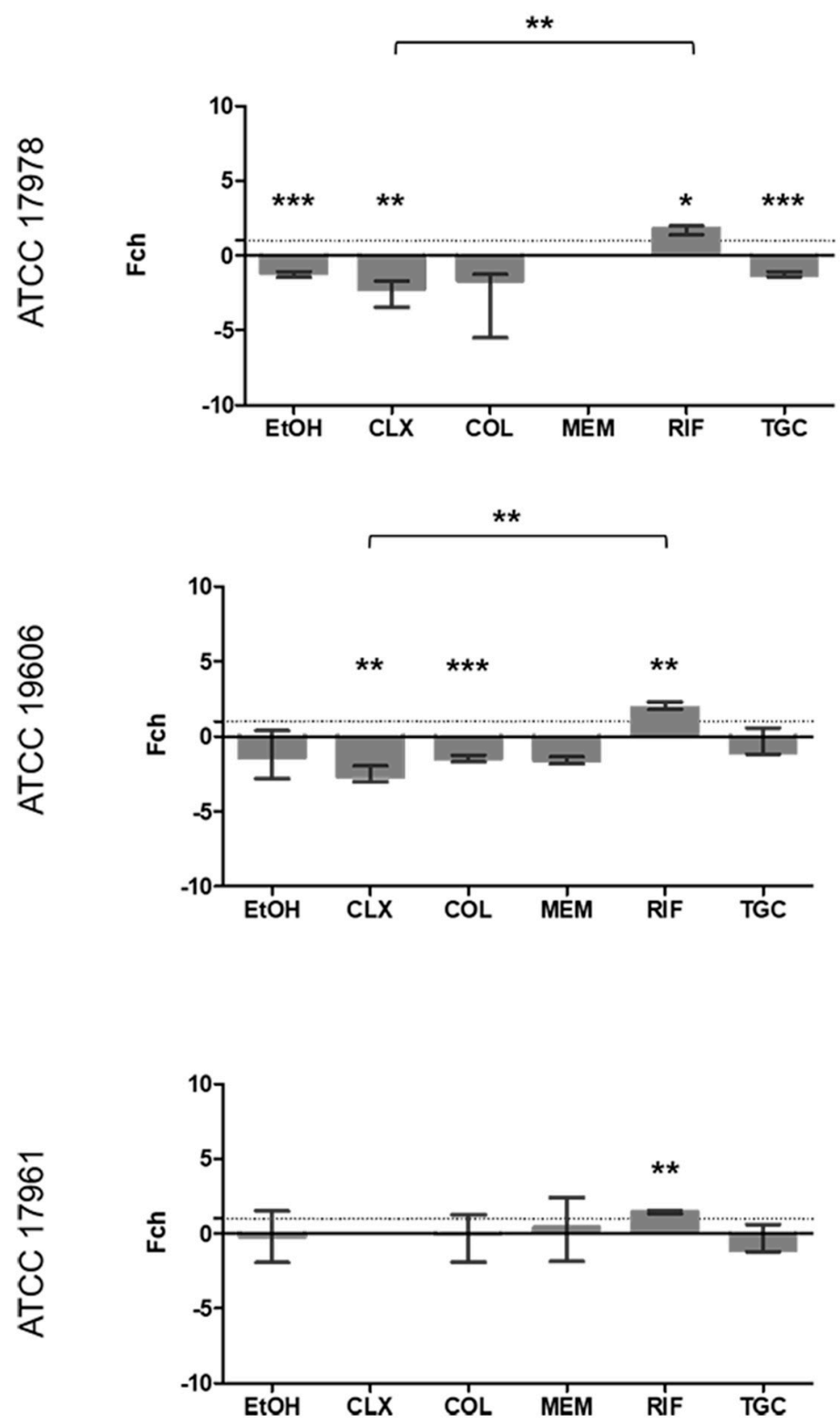

Stationary phase
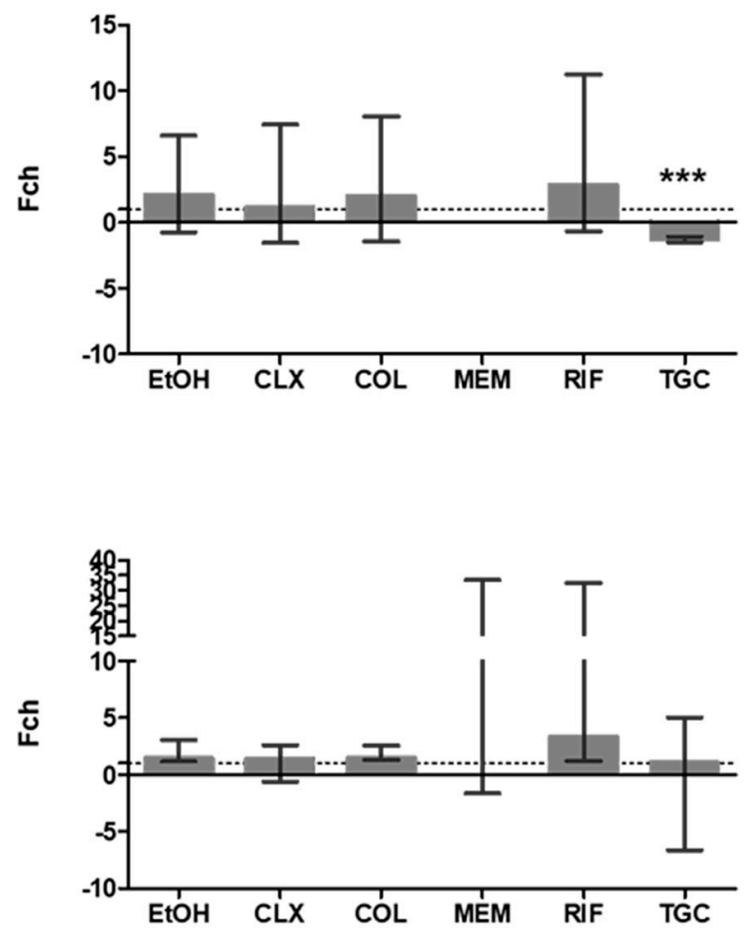

FIGURE 4 | Fold change in adelJK promoter activity in the three strains included in the study calculated at $6 \mathrm{~h}$ (exponential phase) and $24 \mathrm{~h}$ (stationary phase) in the presence of subinhibitory concentrations of antibiotics. The Fold-change represents the difference in luminescence. Values $>1$ are considered as increased expression and values $<1$ as decreased expression. Values = 1 represent no changes in gene expression. There is no representation of groups with no growth $\left(\mathrm{OD}_{600}<0.02\right)$. Brackets represent significant differences between treatments; ${ }^{*} p<0.05,{ }^{* *} p<0.01$ and ${ }^{* \star *} p<0.001$. Colistin $(\mathrm{COL})$, meropenem (MEM), rifampicin (RIF), tigecycline (TGC), chlorhexidine (CLX), and ethanol (EtOH).

of four members of the RND family in different strains of A. baumannii. This approach allowed to screen a large number of combinations of strains, antimicrobials and promoter regions.

Our qualitative data demonstrated basal levels of expression of adeRS and adeIJK whereas expression of adeABC and adeFGH seems to be inducible. Constitutive expression of adeIJK has been previously reported (Damier-Piolle et al., 2008; Leus et al., 2018) whereas this is not the case for adeFGH (Coyne et al., $2010 \mathrm{~b}, 2011$ ) and overexpression of adeABC has been reported as a mechanism of acquired resistance (Magnet et al., 2001). Regardless of their basal expression, our study demonstrates that subinhibitory concentrations of clinically-relevant antimicrobials 


\section{Exponential phase}

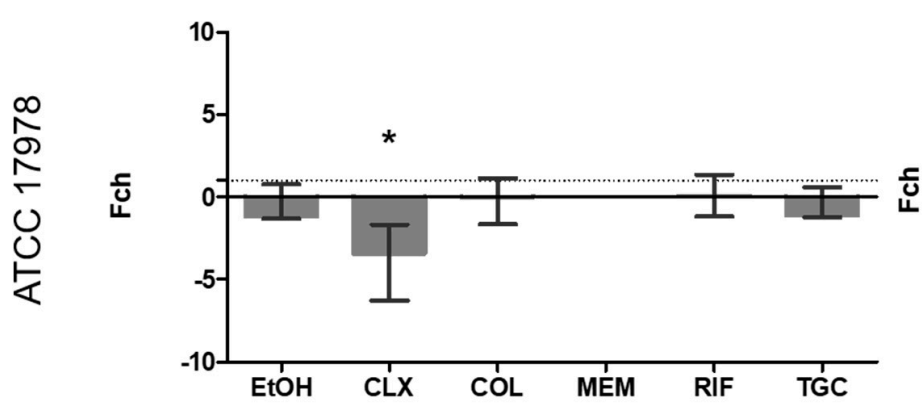

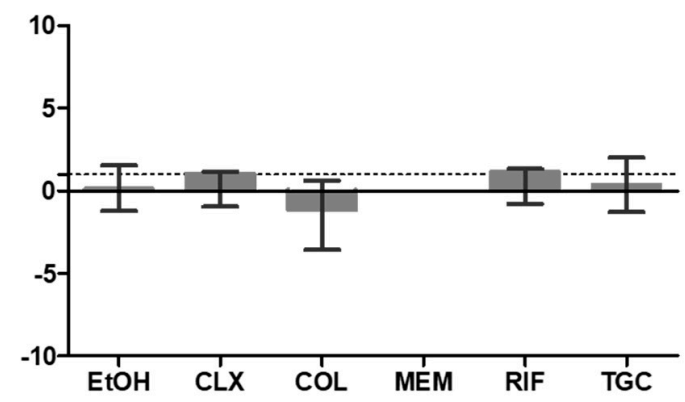

Stationary phase

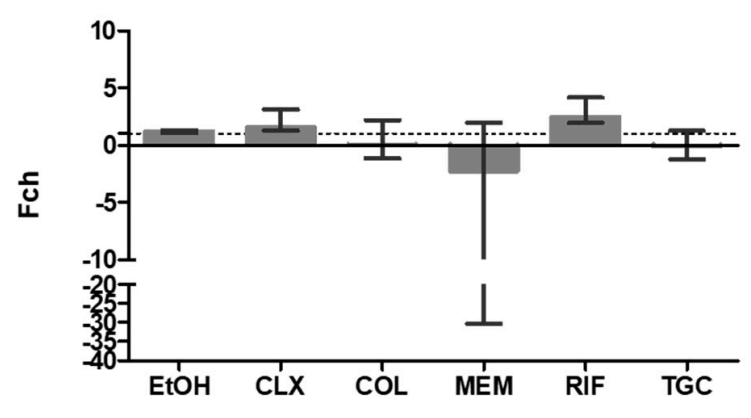

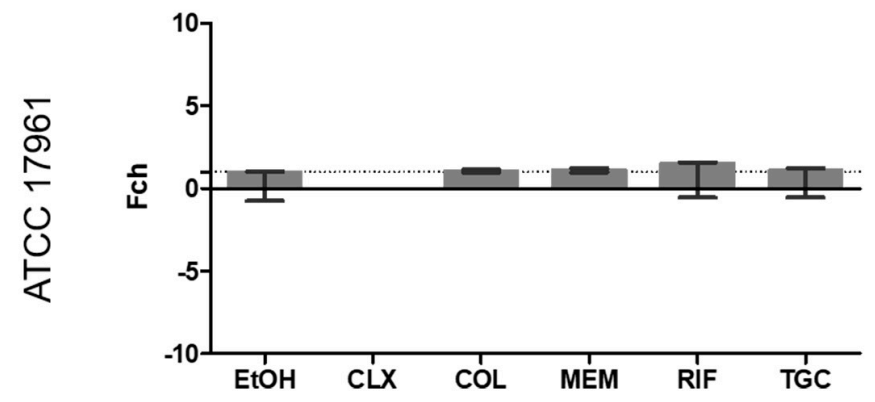

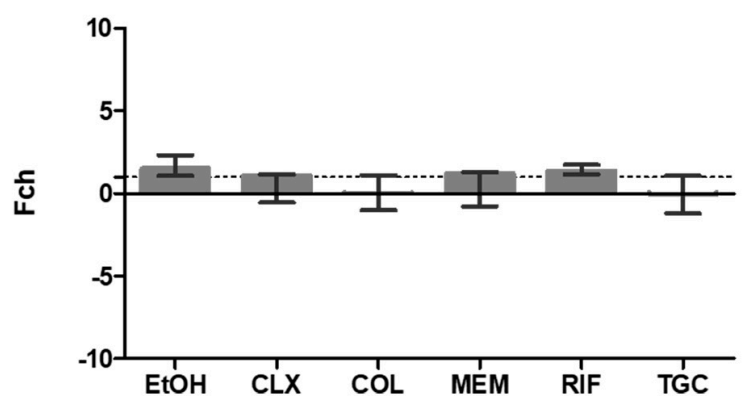

FIGURE 5 | Fold change in adeRS promoter activity in the three strains included in the study calculated at $6 \mathrm{~h}$ (exponential phase) and $24 \mathrm{~h}$ (stationary phase) in the presence of subinhibitory concentrations of antibiotics. The Fold-change represents the difference in luminescence. Values $>1$ are considered as increased expression and values $<1$ as decreased expression. Values = 1 represent no changes in gene expression. There is no representation of groups with no growth $\left(\mathrm{OD}_{600}<0.02\right) ;{ }^{*} p<0.05,{ }^{* *} p<0.01$ and ${ }^{* \star *} p<0.001$. Colistin $(\mathrm{COL})$, meropenem (MEM), rifampicin (RIF), tigecycline (TGC), chlorhexidine (CLX), and ethanol $(\mathrm{EtOH})$.

affect the promoter activity of all the members of the RND family included in this study. However, we have observed that none of the antimicrobials universally affects all pumps, or the regulatory system.

Our data show that EtOH, CLX, and RIF affected adeABC promoter expression. This is in agreement with previous studies (Camarena et al., 2010; Rajamohan et al., 2010; Hassan et al., 2013; Fernández-Cuenca et al., 2015; Lin et al., 2017) mainly using the ATCC 17978 strain (Camarena et al., 2010; Hassan et al., 2013) or clinical isolates (Lin et al., 2017). Whereas previous studies report similar data for disinfectants (Camarena et al., 2010; Hassan et al., 2013), our study is the first to demonstrate that subinhibitory concentrations of RIF affect promoter activity. Notably, we observed that subinhibitory concentration of CLX can induce up to a 130-fold increase in promoter activity of this efflux pump in a particular strain. This is in line with several studies reporting increased expression of this efflux pump when exposed to CLX (Rajamohan et al., 2010; Hassan et al., 2013; Fernández-Cuenca et al., 2015; Lin et al., 2017), including subinhibitory concentrations 


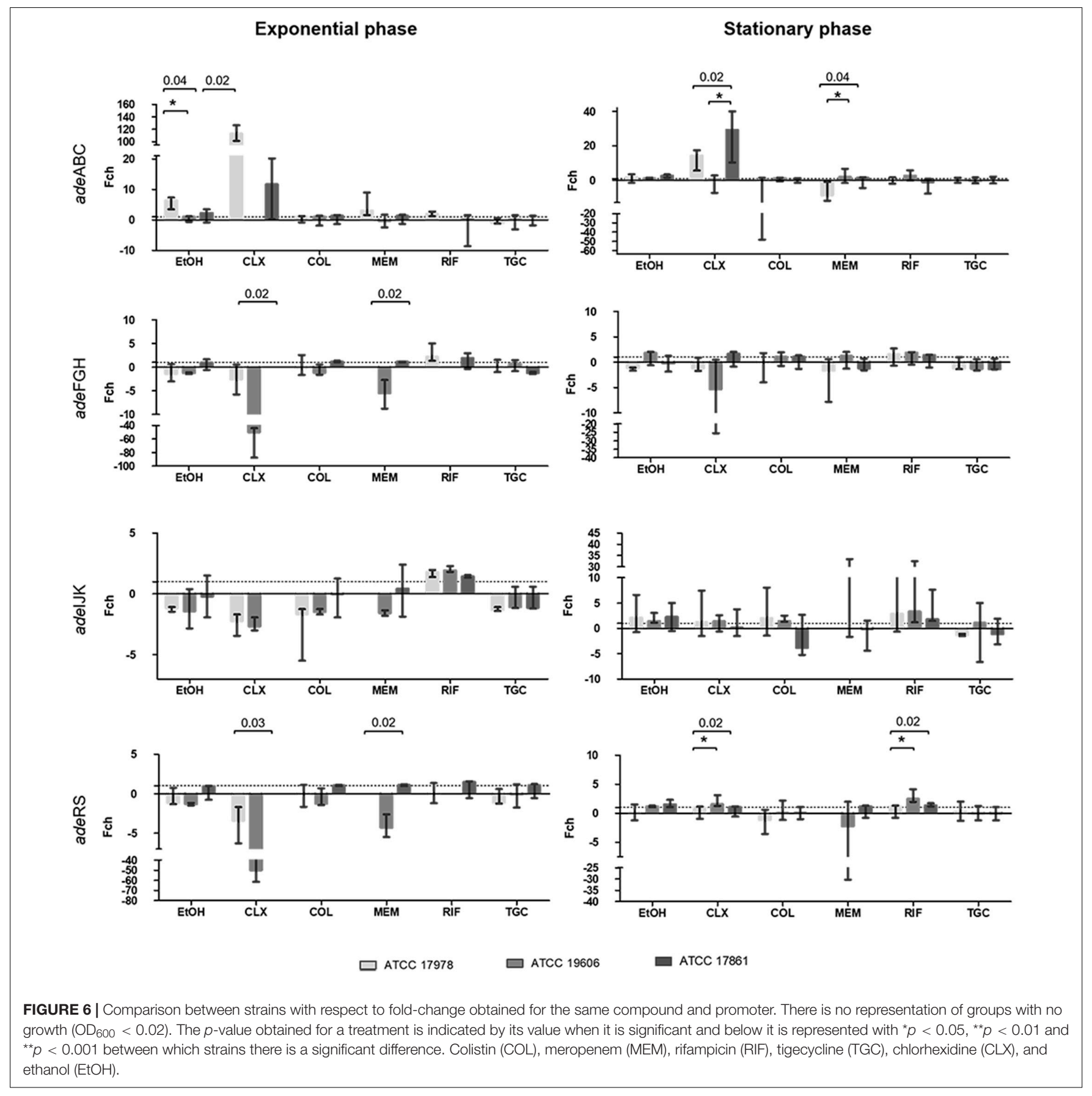

(Hassan et al., 2013; Lin et al., 2017), and supports previous reports indicating that among the tree efflux pumps included in this work, AdeABC appears to be the most affected by this compound (Rajamohan et al., 2010).

With regards to adeFGH, we observed a significantly decreased expression with EtOH, MEM and TGC. On the one hand, this could be due to the fact that AdeFGH is not constitutively expressed and therefore it would not contribute to intrinsic resistance (Coyne et al., 2010a,b). On the other hand, it could be the consequence or a result of other metabolic processes taking place inside the bacterial cell. For instance, EtOH concentrations similar to that used in the present study have been shown to enhance A. baumannii ATCC 17978 growth, and therefore AdeFGH repression may respond to a mechanism to maintain this compound within the cell (Smith et al., 2004). In the case of MEM, downregulation can respond to the presence of other resistance mechanisms that are more efficient, such as carbapenemases (Lee et al., 2010), which is in agreement with studies on other strains of $A$. baumannii showing that $\beta$-lactams are not a substrate of this pump (Coyne et al., 2010b, 2011). 
TABLE 3 | Pearson correlation of promoter activity fold-changes by strain, considering all antimicrobial treatments, in exponential and stationary phases.

\begin{tabular}{|c|c|c|c|c|c|c|}
\hline & \multicolumn{6}{|c|}{ A. baumannii strain } \\
\hline & \multicolumn{2}{|c|}{ ATCC 17978} & \multicolumn{2}{|c|}{ ATCC $19606^{T}$} & \multicolumn{2}{|c|}{ ATCC 17961} \\
\hline & Corr. cof & $p$-value & Corr. cof & $p$-value & Corr. Cof & $p$-value \\
\hline \multicolumn{7}{|l|}{ Exponential phase } \\
\hline adeABC-adeFGH & -0.696 & 0.192 & 0.223 & 0.777 & -0.152 & 0.807 \\
\hline adeABC-adelJK & -0.384 & 0.523 & -0.399 & 0.601 & -0.765 & 0.131 \\
\hline adeABC-adeRS & -0.986 & 0.002 & 0.245 & 0.755 & -0.221 & 0.720 \\
\hline adeFGH-adelJK & 0.773 & 0.125 & 0.858 & 0.063 & 0.667 & 0.219 \\
\hline adeFGH-adeRS & 0.781 & 0.119 & 1.000 & $<0.001$ & 0.540 & 0.348 \\
\hline adelJK-adeRS & 0.467 & 0.428 & 0.851 & 0.068 & 0.321 & 0.598 \\
\hline \multicolumn{7}{|l|}{ Stationary phase } \\
\hline adeABC-adeFGH & 0.236 & 0.652 & 0.789 & 0.062 & 0.577 & 0.231 \\
\hline adeABC-adelJK & -0.111 & 0.859 & 0.858 & 0.029 & 0.050 & 0.924 \\
\hline ade $A B C$-adeRS & 0.844 & 0.072 & -0.376 & 0.463 & -0.084 & 0.875 \\
\hline adeFGH-adelJK & 0.429 & 0.471 & 0.387 & 0.448 & 0.345 & 0.503 \\
\hline adeFGH-adeRS & 0.353 & 0.560 & -0.251 & 0.631 & 0.153 & 0.772 \\
\hline adelJK-adeRS & -0.113 & 0.856 & -0.386 & 0.450 & 0.884 & 0.019 \\
\hline
\end{tabular}

Significant $p$-values $(p \leq 0.05)$ are highlighted in bold.

Conflicting data is reported in the literature concerning the effect of TGC on the adeFGH promoter. Whereas an overexpression of this pump has been associated with reduced susceptibility to TGC (Coyne et al., 2010b), our results revealed downregulation of this promoter when exposed to subinhibitory concentrations of this antibiotic, which is in agreement with a study reported by Navidifar et al. (2019) assessing exposure to concentrations within the MIC range of different strains.

Moderately increased activity of the adeIJK promoter was observed at subinhibitory concentrations of RIF in all three stains tested, which is consistent with the fact that this antibiotic is a substrate of this pump (Damier-Piolle et al., 2008). In contrast, adeIJK expression was significantly reduced by EtOH, CLX, COL, MEM, and TGC. This could be the consequence of redundant metabolic processes within the cell, for instance AdeIJK has been reported to act synergistically with AdeABC to expel TGC (Damier-Piolle et al., 2008). However, it has also been reported that AdeIJK overexpression could be toxic (Damier-Piolle et al., 2008), and therefore its expression may be strictly regulated, in order to maintain non-toxic levels (Coyne et al., 2011). Taken together, our data support previous studies suggesting that this pump may contribute only to intrinsic resistance (Chu et al., 2006; Damier-Piolle et al., 2008; Coyne et al., 2010a). Nonetheless, in our approach the chromosomal copy of adeIJK has not been removed, and therefore, we cannot rule out that by increasing the number of copies of this promoter, the regulator mechanisms of this pump, the TetR transcriptional regulator AdeN (Rosenfeld et al., 2012), had been activated. Also, the different methodologies could explain the conflicting data that exists for some compounds such as MEM or CLX, ranging from no effect on adeIJK expression (Navidifar et al., 2019) to overexpression of this gene (Rajamohan et al., 2010; Leus et al., 2018).
We observed decreased expression of adeRS with subinhibitory concentrations of EtOH, MEM and CLX only during the exponential phase. In this two-component regulatory system, AdeR is the transcriptional regulator and AdeS is a sensor protein kinase, which detects changes in environmental conditions and activates AdeR, which ultimately results in changes in the expression of the AdeABC efflux pump (Magnet et al., 2001; Marchand et al., 2004). In this case, downregulation of adeRS can lead to overexpression of adeABC and thus result in antimicrobial resistance. Whereas we observed that the two disinfectants affected adeRS expression in several strains, the antibiotic MEM affected its expression exclusively in the ATCC $19606^{T}$ strain. To the best of our knowledge, no study has assessed the effect of antimicrobials on the expression of this regulatory system. Nonetheless, point mutations leading to amino acid substitutions in AdeRS have been linked to AdeABC overexpression and carbapenem resistance (Zhang et al., 2017). Despite the fact that the $500 \mathrm{bp}$ upstream of the genes analyzed in this study are very similar between strains, it is possible that the binding sequences of other local or global regulators that naturally affect the expression of adeRS (Wieczorek et al., 2008) have been lost, and that therefore we are not detecting all changes in promoter activity. Further studies to analyze the effect of antimicrobials subinhibitory concentrations in AdeN and AdeL which regulate adeFGH and adeIJK expression, respectively, would be of interest.

Another aspect that our study evidenced is that antimicrobials affect promoter activity in a strain dependent manner. Differences between strains in the expression from adeABC, adeFHG and adeRS promoters were observed at subinhibitory concentrations of EtOH, CLX, MEM and RIF. Strain-dependent responses upon exposure to EtOH, MEM and CLX have been previously reported by Lin et al. (2017). While the former evaluates several genes including adeB, adeJ and adeG and observes this discordance, the latter describes how the control that adeRS exerts on adeABC is strain-specific. Thus, this phenomenon appears to occur with the regulatory system, and according to our data may affect all the pumps studied.

Nonetheless, within the same strain some compounds affected the expression of several promoters. For instance, CLX significantly affected the expression of $a d e A B C$, adeIJK and adeRS in strain ATCC 17978, and we corroborated negative correlation between the former and the latter, which agrees with the fact that AdeRS is a regulatory system of AdeABC. In strain ATCC $19606^{T}$ EtOH and MEM significantly decreased the expression of AdeFGH and AdeRS, and thus positive correlation was found between them in the exponential phase. This may indicate that regulation of RND family members is interconnected. Although few studies have explored the potential association between RND family members, some reports have shown this possibility. For instance inactivation of adeFGH has been reported to trigger overproduction of AdeABC (Leus et al., 2018), and AdeABC and AdeIJK have been shown to contribute synergistically to TGC resistance (Damier-Piolle et al., 2008). Without considering the strain and effect (downregulation or overexpression), we observed that EtOH affected the expression of all the promoters analyzed, and CLX and MEM at least three out of the four 
RND members studied, including the regulatory system and two efflux pumps. In line with this, it was previously reported that the same compound can be exported by several pumps, since efflux pumps represent an important mechanism of resistance as they can expel different compounds with varied structures (Sánchez Díaz, 2003).

Lastly, we observed that even within a strain, a similar pump and compound, differences of the effect were also observed depending on the growth phase. The only compounds that affected adeABC, adeFGH and adeIJK promoters regardless of growth phase were CLX, EtOH and TGC, respectively. In general, most of antimicrobials affected promoter expression during the exponential phase, and fold-changes were higher than those observed in the stationary phase. This may be due to the fact that, during the exponential phase, mechanisms to expel metabolism byproducts are more active. To the best of our knowledge, previous studies analyzing the effects of antimicrobials on RND members have focused either on the exponential phase (Camarena et al., 2010; Coyne et al., 2010a,b; Hassan et al., 2013; Lin et al., 2017) or stationary phase (Navidifar et al., 2019) but none compared both. Our study complements previous reports indicating that the effect of subinhibitory concentrations on promoter expression was growth phase dependent in some cases, which may have relevance for antimicrobial therapy, for example, in the context of biofilms.

\section{CONCLUSION}

Altogether, the results obtained in this work support the idea that subinhibitory concentrations of antimicrobials have an effect on the expression of both efflux pumps and the regulatory systems of the RND family in $A$. baumannii. Future work aimed at defining the effect of antimicrobials on other regulatory elements involved in efflux pump regulation (e.g., AdeN), and the effect of additional clinically relevant antibiotics (e.g., third generation cephalosporins) using this system may be of interest for obtaining a more complete picture of efflux pump regulation. Furthermore, the fact that not all antibiotics produce a significant effect on all pumps is evident. Moreover, the effect they produce was shown to be highly dependent upon the strain, promoter and growth phase, suggesting that the gene content of the tested strain influences promotor activity. Since the effect of the compounds is not universal, it seems that the acquisition of resistance depends on a combination of several mechanisms, which requires further study.

\section{REFERENCES}

Ardebili, A., Lari, A. R., and Talebi, M. (2014). Correlation of ciprofloxacin resistance with the AdeABC efflux system in Acinetobacter baumannii clinical isolates. Ann. Lab. Med. 34, 433-438. doi: 10.3343/alm.2014.34. 6.433

Boucher, H. W., Talbot, G. H., Bradley, J. S., Edwards, J. E., Gilbert, D., Rice, L. B., et al. (2009). Bad bugs, no drugs: no ESKAPE! An update from the

\section{DATA AVAILABILITY STATEMENT}

The raw data supporting the conclusions of this article will be made available by the authors upon request, without undue reservation.

\section{AUTHOR CONTRIBUTIONS}

SPMG performed antimicrobials susceptibility testing, plasmid construction, quantitative analysis of expression, analyzed data, drafted the manuscript, and revised the final version. AT performed qualitative analysis of expression and revised the final version of the manuscript. ML-S assisted in plasmid construction, quantitative and qualitative analysis of expression, data analysis, supervised the study and drafted the manuscript. MJM conceived and supervised the study, revised the manuscript and granted funding. All authors contributed to the article and approved the submitted version.

\section{FUNDING}

AT is supported by the Garantía Juvenil Program of the Comunidad Autonoma de Madrid and ML-S is supported by the Sara Borrell Program of the Instituto de Salud Carlos III. MJM is supported by grants from the Instituto de Salud Carlos III (MP 516/19 and MPY 380/18).

\section{ACKNOWLEDGMENTS}

We would like to thank Paolo Visca (University Roma Tre) for kindly providing pLPV1Z plasmid. We would also like to thank Javier García Pérez for helping with luminometry assays (AIDS Immunopathology Unit, National Center for Microbiology, Instituto de Salud Carlos III), Javier Pardos Bartolomé (Veterinary Unit, National Center for Microbiology, Instituto de Salud Carlos III) for assistance with bioluminescence images analyses, and Antonio Javier Martín Galiano (Intrahospital Infections Laboratory, National Center for Microbiology, Instituto de Salud Carlos III) for promoter sequences analyses.

\section{SUPPLEMENTARY MATERIAL}

The Supplementary Material for this article can be found online at: https://www.frontiersin.org/articles/10.3389/fmicb. 2021.780201/full\#supplementary-material

infectious diseases society of America. Clin. Infect. Dis. 48, 1-12. doi: 10.1086/5 95011

Camarena, L., Bruno, V., Euskirchen, G., Poggio, S., and Snyder, M. (2010). Molecular mechanisms of ethanol-induced pathogenesis revealed by RNAsequencing. PLoS Pathog. 6:e1000834. doi: 10.1371/journal.ppat.1000834

Chu, Y. W., Chau, S. L., and Houang, E. T. S. (2006). Presence of active efflux systems AdeABC, AdeDE and AdeXYZ in different Acinetobacter genomic DNA groups. J. Med. Microbiol. 55, 477-478. doi: 10.1099/jmm.0.46433-0 
CLSI (2012). Performance Standards for Antimicrobial Susceptibility Testing; Twenty-Second Informational Supplement. CLSI Document M100-S22. Wayne, PA: Clinical and Laboratory Standards Institute.

CLSI (2017). Performance Standards for Antimicrobial Susceptibility Testing; Twenty-Seventh Informational Supplement. CLSI Document M100-S27-. Wayne, PA: Clinical Laboratory Standards institute.

Coyne, S., Courvalin, P., and Périchon, B. (2011). Efflux-mediated antibiotic resistance in Acinetobacter spp. Antimicrob. Agents Chemother. 55, 947-953. doi: 10.1128/AAC.01388-10

Coyne, S., Guigon, G., Courvalin, P., and Périchon, B. (2010a). Screening and quantification of the expression of antibiotic resistance genes in Acinetobacter baumannii with a microarray. Antimicrob. Agents Chemother. 54, 333-340. doi: 10.1128/AAC.01037-09

Coyne, S., Rosenfeld, N., Lambert, T., Courvalin, P., and Périchon, B. (2010b). Overexpression of resistance-nodulation-cell division pump AdeFGH confers multidrug resistance in Acinetobacter baumannii. Antimicrob. Agents Chemother. 54, 4389-4393. doi: 10.1128/AAC.00 155-10

Damier-Piolle, L., Magnet, S., Brémont, S., Lambert, T., and Courvalin, P. (2008). AdeIJK, a resistance-nodulation-cell division pump effluxing multiple antibiotics in Acinetobacter baumannii. Antimicrob. Agents Chemother. 52, 557-562. doi: 10.1128/AAC.00732-07

Dijkshoorn, L., Nemec, A., and Seifert, H. (2007). An increasing threat in hospitals: multidrug-resistant Acinetobacter baumannii. Nat. Rev. Microbiol. 5, 939-951. doi: 10.1038/nrmicro1789

Falagas, M. E., and Rafailidis, P. I. (2007). Attributable mortality of Acinetobacter baumannii: no longer a controversial issue. Crit. Care 11:134. doi: 10.1186/ cc5911

Fernández-Cuenca, F., Tomás, M., Caballero-Moyano, F. J., Bou, G., MartínezMartínez, L., Vila, J., et al. (2015). Reduced susceptibility to biocides in Acinetobacter baumannii: association with resistance to antimicrobials, epidemiological behaviour, biological cost and effect on the expression of genes encoding porins and efflux pumps. J. Antimicrob. Chemother. 70, 3222-3229. doi: $10.1093 / \mathrm{jac} / \mathrm{dkv} 262$

Fournier, P. E., Vallenet, D., Barbe, V., Audic, S., Ogata, H., Poirel, L., et al. (2006). Comparative genomics of multidrug resistance in Acinetobacter baumannii. PLoS Genet. 2:e7. doi: 10.1371/journal.pgen.0020007

Hassan, K. A., Jackson, S. M., Penesyan, A., Patching, S. G., Tetu, S. G., Eijkelkamp, B. A., et al. (2013). Transcriptomic and biochemical analyses identify a family of chlorhexidine efflux proteins. Proc. Natl. Acad. Sci. U.S.A. 110, 20254-20259. doi: $10.1073 /$ pnas. 1317052110

Helling, R. B., Janes, B. K., Kimball, H., Tran, T., Bundesmann, M., Check, P., et al. (2002). Toxic waste disposal in Escherichia coli. J. Bacteriol. 184, 3699-3703. doi: 10.1128/jb.184.13.3699-3703.2002

Karakonstantis, S., Kritsotakis, E. I., and Gikas, A. (2020). Treatment options for K. pneumoniae, $P$. aeruginosa and A. baumannii co-resistant to carbapenems, aminoglycosides, polymyxins and tigecycline: an approach based on the mechanisms of resistance to carbapenems. Infection 48, 835-851. doi: 10.1007/ s15010-020-01520-6

Kyriakidis, I., Vasileiou, E., Pana, Z. D., and Tragiannidis, A. (2021). Acinetobacter baumannii antibiotic resistance mechanisms. Pathogens 10:373, doi: 10.3390/ pathogens 10030373

Lari, A. R., Ardebili, A., and Hashemi, A. (2018). AdeR-AdeS mutations \& overexpression of the AdeABC efflux system in ciprofloxacin-resistant Acinetobacter baumannii clinical isolates. Indian J. Med. Res. 147, 413-421. doi: 10.4103/ijmr.ijmr_644_16

Lee, Y., Yum, J. H., Kim, C. K., Yong, D., Jeon, E. H., Jeong, S. H., et al. (2010). Role of OXA-23 and AdeABC efflux pump for acquiring carbapenem resistance in an Acinetobacter baumannii strain carrying the blaOXA-66 gene. Ann. Clin. Lab. Sci. 40, 43-48.

Leung, W. S., Chu, C. M., Tsang, K. Y., Lo, F. H., Lo, K. F., and Ho, P. L. (2006). Fulminant community-acquired Acinetobacter baumannii pneumonia as a distinct clinical syndrome. Chest 129, 102-109. doi: 10.1378/chest.129.1. 102

Leus, I. V., Weeks, J. W., Bonifay, V., Smith, L., Richardson, S., and Zgurskaya, H. I. (2018). Substrate specificities and efflux efficiencies of RND efflux pumps of Acinetobacter baumannii. J. Bacteriol. 200:e00049-18, doi: 10.1128/JB. 00049-18
Lin, F., Xu, Y., Chang, Y., Liu, C., Jia, X., and Ling, B. (2017). Molecular characterization of reduced susceptibility to biocides in clinical isolates of Acinetobacter baumannii. Front. Microbiol. 8:1836. doi: 10.3389/fmicb.2017. 01836

Lin, M. F., and Lan, C. Y. (2014). Antimicrobial resistance in Acinetobacter baumannii: from bench to bedside. World J. Clin. Cases 2, 787-814.

Lucidi, M., Visaggio, D., Prencipe, E., Imperi, F., Rampioni, G., Cincotti, G., et al. (2019). New shuttle vectors for real-time gene expression analysis in multidrugresistant Acinetobacter species: in vitro and in vivo responses to environmental stressors. Appl. Environ. Microbiol. 85, e01334-19, doi: 10.1128/AEM.01334-19

Magnet, S., Courvalin, P., and Lambert, T. (2001). Resistance-nodulationcell division-type efflux pump involved in aminoglycoside resistance in Acinetobacter baumannii strain BM4454. Antimicrob. Agents Chemother. 45, 3375-3380. doi: 10.1128/AAC.45.12.3375-3380.2001

Marchand, I., Damier-Piolle, L., Courvalin, P., and Lambert, T. (2004). Expression of the RND-type efflux pump AdeABC in Acinetobacter baumannii is regulated by the AdeRS two-component system. Antimicrob. Agents Chemother. 48, 3298-3304. doi: 10.1128/AAC.48.9.3298-3304.2004

McConnell, M. J., Actis, L., and Pachón, J. (2013). Acinetobacter baumannii: human infections, factors contributing to pathogenesis and animal models. FEMS Microbiol. Rev. 37, 130-155. doi: 10.1111/j.1574-6976.2012.00344.x

Nang, S. C., Azad, M. A. K., Velkov, T., Zhou, Q. T., and Li, J. (2021). Rescuing the last-line polymyxins: achievements and challenges. Pharmacol. Rev. 73, 679-728. doi: 10.1124/pharmrev.120.000020

Navidifar, T., Amin, M., and Rashno, M. (2019). Effects of sub-inhibitory concentrations of meropenem and tigecycline on the expression of genes regulating pili, efflux pumps and virulence factors involved in biofilm formation by Acinetobacter baumannii. Infect. Drug Resist. 12, 1099-1111. doi: 10.2147/ IDR.S199993

Nikaido, H. (1994). Prevention of drug access to bacterial targets: permeability barriers and active efflux. Science 264, 382-388.

Opazo, A. C., Mella, S. M., Domínguez, M. Y., Bello, H. T., and González, G. R. (2009). Multi-drug efflux pumps and antibiotic resistance in Acinetobacter baumannii. Rev. Chilena Infectol. 26, 499-503.

Picazo, J. J. (2000). Métodos Básicos Para el Estudio de la Sensibilidad a Antimicrobianos. Procedimientos en Microbiología. SEIMC. Available online at: https://www.seimc.org/contenidos/documentoscientificos/ procedimientosmicrobiologia/seimc-procedimientomicrobiologia11.pdf (accessed July 5, 2019).

Poole, K. (2002). Outer membranes and efflux: the path to multidrug resistance in gram-negative bacteria. Curr. Pharm. Biotechnol. 3, 77-98.

Rajamohan, G., Srinivasan, V. B., and Gebreyes, W. A. (2010). Novel role of Acinetobacter baumannii RND efflux transporters in mediating decreased susceptibility to biocides. J. Antimicrob. Chemother. 65, 228-232. doi: 10.1093/ jac/dkp427

Rosenfeld, N., Bouchier, C., Courvalin, P., and Périchon, B. (2012). Expression of the resistance-nodulation-cell division pump AdeIJK in Acinetobacter baumannii is regulated by AdeN, a TetR-type regulator. Antimicrob. Agents Chemother. 56, 2504-2510. doi: 10.1128/AAC.06422-11

Salehi, B., Ghalavand, Z., Yadegar, A., and Eslami, G. (2021). Characteristics and diversity of mutations in regulatory genes of resistance-nodulation-cell division efflux pumps in association with drug-resistant clinical isolates of Acinetobacter baumannii. Antimicrob. Resist. Infect. Control 10:53. doi: 10.1186/s13756-02100924-9

Sánchez Díaz, P. (2003). [MDR efflux pumps and antimicrobial resistance]. Rev. Esp. Quimioter. 16, 172-187.

Smith, M. G., Des Etages, S. G., and Snyder, M. (2004). Microbial synergy via an ethanol-triggered pathway. Mol. Cell. Biol. 24, 3874-3884. doi: 10.1128/MCB. 24.9.3874-3884.2004

Taconnelli, E., and Magrini, N. (2017). Mundial Priority List of Antibiotic-Resistant Bacteria to Guide Research, Discovery, and Development of New Antibiotics. Geneva: world health organization.

The European Committee on Antimicrobial Susceptibility Testing (2021). Breakpoint Tables for Interpretation of MICs and Zone Diameters. Version 11.0, Available online at: http://www.eucast.org. (accessed May 31, 2021).

Vila, J., Martí, S., and Sánchez-Céspedes, J. (2007). Porins, efflux pumps and multidrug resistance in Acinetobacter baumannii. J. Antimicrob. Chemother. 59, 1210-1215. doi: $10.1093 / \mathrm{jac} / \mathrm{dkl} 509$ 
Wieczorek, P., Sacha, P., Hauschild, T., Zórawski, M., Krawczyk, M., and Tryniszewska, E. (2008). Multidrug resistant Acinetobacter baumannii-the role of AdeABC (RND family) efflux pump in resistance to antibiotics. Folia Histochem. Cytobiol. 46, 257-267. doi: 10.2478/v10042-0080056-x

Yoon, E. J., Courvalin, P., and Grillot-Courvalin, C. (2013). RND-type efflux pumps in multidrug-resistant clinical isolates of Acinetobacter baumannii: major role for AdeABC overexpression and AdeRS mutations. Antimicrob. Agents Chemother. 57, 2989-2995. doi: 10.1128/AAC.02556-12

Zhang, Y., Li, Z., He, X., Ding, F., Wu, W., Luo, Y., et al. (2017). Overproduction of efflux pumps caused reduced susceptibility to carbapenem under consecutive imipenem-selected stress in Acinetobacter baumannii. Infect. Drug Resist. 11, 457-467. doi: 10.2147/IDR.S151423

Conflict of Interest: MJM is founder and stockholder of the biotechnology spin-off company Vaxdyn, which develops vaccines for infections caused by MDR bacteria. Vaxdyn had no role in the elaboration of this manuscript.
The remaining authors declare that the research was conducted in the absence of any commercial or financial relationships that could be construed as a potential conflict of interest.

Publisher's Note: All claims expressed in this article are solely those of the authors and do not necessarily represent those of their affiliated organizations, or those of the publisher, the editors and the reviewers. Any product that may be evaluated in this article, or claim that may be made by its manufacturer, is not guaranteed or endorsed by the publisher.

Copyright $(2021$ Prieto Martin Gil, Tajuelo, López-Siles and McConnell. This is an open-access article distributed under the terms of the Creative Commons Attribution License (CC BY). The use, distribution or reproduction in other forums is permitted, provided the original author(s) and the copyright owner(s) are credited and that the original publication in this journal is cited, in accordance with accepted academic practice. No use, distribution or reproduction is permitted which does not comply with these terms. 